\title{
ANÁLISIS LONGITUDINAL DE LA COMORBILIDAD ENTRE DEPRESIÓN Y AGRESIÓN: CRONICIDAD Y SEVERIDAD EN SUJETOS DE 11 A 13 AÑOS
}

\section{LONGITUDINAL ANALYSIS OF COMORBIDITY BETWEEN DEPRESSION AND AGGRESSION: CHRONICITY AND SEVERITY IN INDIVIDUALS 11 TO 13 YEARS}

\author{
Rodolfo Gordillo*, Victoria del Barrio** y Miguel Ángel Carrasco*** \\ *Doctor en Psicología. Profesor Adjunto de la Universidad a Distancia de Madrid (UDIMA). \\ Camino de la Fonda, 20 - 28400 Collado Villalba. Madrid. E-Mail: rodolfo.gordillo@udima.es \\ **Doctora en Psicología. Profesora Titular de Evaluación Psicológica en la Facultad de \\ Psicología de la Universidad Nacional de Educación a Distancia (UNED). \\ ***Doctor en Psicología y MA en Terapia de Conducta. Profesor Titular de la Facultad de \\ Psicología de la Universidad Nacional de Educación a Distancia (UNED).
}

\section{REsumen}

La comorbilidad entre depresión y agresión infanto-adolescente ha sido contrastada básicamente a un nivel estadístico. Sin embargo, pocos estudios han comprobado de manera longitudinal otra de las premisas fundamentales que permite establecer la comorbilidad: la sinergia, es decir, la acción conjunta de dos o más trastornos que se traduce en una severidad y cronicidad superior a las generadas por la suma de los efectos individuales. Mediante un diseño longitudinal a lo largo de 3 años se examinó la severidad y cronicidad de un grupo de sujetos comórbidos depresivos - agresivos. Se analizaron los niveles de depresión y agresión, así como el papel del sexo en estas relaciones. La evaluación se realizó a través de autoinformes en una muestra de 525 sujetos de edades comprendidas entre los 11 y 13 años. La muestra fue aleatoriamente seleccionada de diferentes colegios de la Comunidad de Madrid (España). Los resultados muestran que la depresión y agresión se relacionan de manera estadísticamente significativa. Los sujetos comórbidos exhiben una cronicidad de mayor duración en las respuestas agresivas que en las depresivas.
La severidad en los sujetos comórbidos es mayor en las respuestas agresivas de tipo total y verbal, así como en las depresivas. El sexo no ejerce ningún efecto significativo. Se concluye que los datos indican la importancia de explorar la comorbilidad con diferentes tipos de agresión, especialmente la verbal, y en diferentes grupos de edad. Se discute la necesidad de atender a los síntomas subyacentes al trastorno principal en afectados de larga duración, ya que a la expresión de uno de los trastornos puede subyacer la cronicidad asociada a la comorbilidad con importantes repercusiones para su diagnóstico y tratamiento.

Palabras clave: Comorbilidad; Depresión; Agresión física y verbal; Infantil; Adolescente.

\section{ABSTRACT}

The comorbidity between depression and aggression in children and adolescents is an issue that has sparked controversy as its existence has important implications for the validity of the classification systems, for the establishment of 
explanatory models, the design of new treatments as well as in the final outcome which the comorbid person presents in these in comparison with conventional treatments. In addition, the prevalence rates in depressive-aggressive comorbidity are significant, oscillating between 7.97 and $10.91 \%$. Therefore, the study of comorbidity becomes necessary and the lack of studies focusing on the subject comes as a surprise. Perhaps one of the main problems concerning the research of comorbidity lies in the fact that most of the studies do not validate the clinical criteria that focuses on the principle of synergy, as Clark and Watson had established in Tripartite model, since doing so would require longitudinal studies measuring the effects of a commorbid disorder in contrast to those where there is no comorbidity to be found. In addition to clinical judgment, the Tripartite model demands a further statistic indicating the existence of a significant correlation between those synergistic disorders, which is the one on which most studies have been focusing. This type of results, based solely on clinical judgment, mainly through cross-sectional studies, has not allowed to implement another type of results, as suggested by the Tripartite model, that would have helped to establish clinical and diagnostic criteria through dimensional self-assessment tools which are closer to a reality in child and adolescent assessment where comorbidity is rather the rule than the exception. Therefore, that kind of study, using a longitudinal design over a period of three years, will help expand those cross-sectional ones, which will allow for examining whether there is a significant association, as well as a synergy that attests to the existence of an aggressive-depressive comorbidity on both, statistical and clinical level. In order to do so this study examined the severity and chronicity of a group of comorbid depressiveaggressive persons. In addition, the levels of depression and aggression and the role of sex within these relationships were analyzed. The sample, randomly selected from different schools in the Community of Madrid (Spain), comprised 525 individuals ranging from 11 to 13 years old, $58.9 \%$ female, the family structure was mostly biparental (92\%) and the vast majority belonged to a middle class environment (85\%). Depression and aggression were assessed through specific types of self-report questionnaires for children and adolescents, both in their Spanish version. The results support the statistical basis, since depression and aggression exhibit a significant positive and moderate association ranging between .11 and .24 , in which the fact that the found association was higher within verbal aggression than in the physical one among all ages with the exception of the group of subjects to testing aged 12 needs to be stressed. With regards to the clinical criteria, the results provide partial support, as comorbid subjects exhibit a longer duration in chronic aggressive symptoms than those other groups affected only by depression or aggression, while they are similar to those of merely depressive subjects in the symptoms indicating a depression. Regarding severity, comorbid subjects show a higher level within the field of totally and verbally aggressive symptoms, as well as in the depressive ones which makes their physical aggression comparable to the one found in the test group of total aggression, hence, the data partially support the clinical standard. The tested person's sex, however, is of no significant meaning for the outcome. In conclusion, the data indicates the importance of exploring the comorbidity of different types of aggression, particularly the verbal one, and within different age groups. The need for addressing the underlying primary disorder symptoms in long-term affected patients is discussed, since chronic comorbidity may underlie the expression of one of the disorders and entail important implications for diagnosis and treatment.

Key words: Comorbidity; Depression; Aggression physical and verbal; Child; Adolescent.

La comorbilidad es un tema controvertido que ha suscitado abundante investigación en la última década (Angold, Costello \& Erkanli, 1999; Frías, Carrasco, Fernández, García, A. \& García, B., 2009; Lewinsohn, Rohde, Seeley \& Hops, 1991; Pössel, Seemann \& Hautzinger, 2008; Teesson, Degenhardt, Proudfoot, Hall \& Lynskey, 2005; Wakefield, Schmitz \& Baer, 2010). 
La relevancia de su estudio se justifica, al menos por cuatro razones: (1) su implicación para la validez de los sistemas de clasificación (Higa-McMillan, Smith, Chorpita \& Hayashi, 2008; Lilienfeld, 2003; Seeley, 2002; Wolff \& Ollendick, 2006); (2) su contribución en el establecimiento de modelos explicativos con mejores ajustes a la realidad (Lilienfeld, 2003; Seeley, 2002); (3) para mejorar el diseño de nuevos y más acertados tratamientos (Angold et al., 1999; Ezpeleta, Granero \& Domenech, 2005; Jensen, 2003; Keiley, Lofthouse, Bates, Dodge \& Pettit, 2003; Seeley, 2002; Subbarao et al., 2008; Wolff \& Ollendick, 2006) y (4) por las repercusiones de la comorbilidad, en términos de gravedad, respuestas al tratamiento y cronicidad (Beyers \& Loeber, 2003; Clark \& Watson, 1991; Clark, Watson \& Reynolds, 1995; Teesson et al., 2005; Youngstrom, Findling \& Calabrese, 2003). A estas razones, se añaden las tasas de prevalencia halladas en población de 9 a 12 años de edad, las cuales han oscilado entre un 7.97 y un $10.91 \%$ (Quiggle, Garber, Panak \& Dodge, 1992; Rudolph \& Clark, 2001). A pesar de estas preocupantes tasas, pocas investigaciones en población española han estudiado la comorbilidad, y menos aún en lo que se refiere a la agresión y a la depresión.

Uno de los modelos más importantes para el estudio de la comorbilidad es el Modelo Tripartito de Clark y Watson (1991), el cual ha sido extensamente validado tanto en población adulta (de Beurs, den HollanderGijsman, Helmich \& Zitman, 2007; Watson et al., 1995), como infantil y adolescente (Chorpita, 2002; Lambert, McCreary, Joiner, Schmidt \& Ialongo, 2004; Phillips \& Lonigan, 2002; Yang, Hong, Joung \& Kim, 2006). De acuerdo con este modelo, para establecer la comorbilidad entre dos trastornos han de cumplirse dos criterios, a saber: uno estadístico y otro clínico. El primero, es que ambos trastornos correlacionen significativamente y el segundo, que aparezca una sinergia en tal asociación, es decir, la acción de dos o más trastornos debe generar una severidad y cronicidad superior a la suma de los efectos individuales. Respecto al primero de estos criterios, en lo que se refiere a la comorbilidad entre agresión y depresión, distintos trabajos han aportado clara evidencia sobre la relación de estas dos alteraciones más allá del azar, reflejando el estrecho grado de covariación entre ambas cuando alguna de ellas aumenta su nivel, con una asociación entre ambos trastornos que oscila entre .34 y .67. Además, la comorbilidad encontrada ha mostrado una robustez multi-informante y multi-criterio, es decir, se ha puesto de manifiesto tanto a través de diferentes fuentes de información (e.g., auto-informe, nominación a través de pares) (Messer \& Gross, 1994), como por criterios dimensionales y categóricos (Angold \& Costello, 1993; Kazdin, French, Unis \& Esveldt-Dawson, 1983; Lefkowitz \& Tesiny, 1985; Saylor, Finch, Furey, Baskin \& Kelly, 1984). Estas evidencias sugieren que ambos trastornos comparten en su etiología ciertas características sintomáticas específicas, ofreciendo una respuesta distinta que explica su comorbilidad. A pesar de estas primeras y claras evidencias, la interacción que se produce entre ambos trastornos apenas ha sido estudiada, y cuando se hizo fue estudiando la agresión como parte de una dimensión más global etiquetada como desórdenes de conducta (Hale, Van Der Valk, Engels \& Meeus, 2005). Esta simplificación, como han señalado Rowe y sus colaboradores, es un error, dado que supone agrupar conductas con identificadores y características propias, tanto en su manifestación como en su evolución bajo una misma denominación (Rowe, Maughan \& Eley, 2006; Rowe, Rijsdijk, Maughan, Hosang \& Eley, 2008), menospreciando la importancia que la comorbilidad entre depresión y agresión pueda tener en términos de clasificación, diagnóstico y tratamiento.

En relación con el segundo de los criterios mencionados, la sinergia derivada de la asociación de dos trastornos, estudios posteriores al de Clark y Watson (1991), no sólo han encontrado en sus resultados mayor severidad, cronicidad y peor pronóstico al tratamiento cuando dos trastornos coexis- 
ten significativamente, sino que esta coexistencia cuando ocurre en la infancia o adolescencia tiene además, un vínculo y significación clínica superior al hallado en la población adulta, ya que en población infanto-juvenil, la comorbilidad es más la regla que la excepción (Evans \& Frank, 2004). Por ello, su estudio en etapas previas a la adulta supone indagar nuevas claves sobre sus orígenes (Hammen \& Compas, 1994), así como la consecución de avances metodológicos y conceptuales que nos ayudarán al entendimiento de la psicopatología infantil y adolescente (Jensen, 2003).

Teniendo estas consideraciones en cuenta, el estudio de la comorbilidad entre dos de los trastornos más prevalentes en la población infantil y adolescente, como son la depresión y la agresión, se torna de gran relevancia para establecer estrategias de intervención más exitosas, ayudar a establecer un diagnóstico más correcto $y$, por ende, una clasificación más acorde a las propiedades sintomáticas de tal comorbilidad.

Los escasos estudios que han investigado las características diferenciales de la comorbilidad depresivo-agresiva en la población infantil y adolescente han destacado como características de esta población, las siguientes:

1.- Sufren los síntomas de ambos desórdenes puros, pero con niveles superiores de malestar general al de los sujetos no comórbidos (Rudolph \& Clark, 2001).

2.- Presentan las características cognitivas de ambos trastornos (Quiggle et al., 1992),

3.- Desaparecen las diferencias entre sexos en la agresión tanto física como verbal (Knox, King, Hanna, Logan \& Ghaziuddin, 2000; Messer \& Gross, 1994).

4.- Los síntomas de agresión y depresión comparten una estructura subyacente de primer orden, que explica el solapamiento encontrado entre ambos trastornos (Hogansen, 2004).
Por todo lo referido anteriormente, se comprende la dificultad que entraña el establecimiento de la comorbilidad, pero también la necesidad y relevancia de su investigación. Este estudio tiene como objetivo general, explorar la existencia de comorbilidad entre agresión y depresión en población infanto-adolescente, su expresión, curso y severidad. Además, este estudio recorre un período especialmente sensible en el desarrollo del individuo (desde los 11 a los 13 años) en el que tiene que enfrentarse a una serie de retos sociales y cambios fisiológicos tales como:

a.- la consolidación del yo (Harter, 1998; Harter \& Monsour, 1992; Serrano, Godás, Rodríguez \& Mirón, 1996),

b.- los cambios hormonales (Cyranowski, Frank, Young \& Shear, 2000),

c.- la toma de decisiones académicas importantes (Serrano et al., 1996),

e.- el afrontamiento de la identidad y orientación sexual (Serrano et al., 1996) $\mathrm{y}$

d.- profundos conflictos emocionales relacionados con el amor, los amigos y la familia (Crockett \& Randall, 2006; Steinberg \& Morris, 2001), cuyo desajuste da lugar a problemas depresivos y agresivos.

Por todo ello, la investigación realizada teniendo en cuenta este tramo evolutivo particularmente sensible, persiguió los siguientes objetivos específicos:

1.- Explorar el grado de comorbilidad entre depresión y agresión en una población española.

2.- Estudiar las consecuencias que sufren los sujetos comórbidos en su respuesta depresiva y agresiva.

3.- Explorar el nivel de intensidad de las respuestas depresivas y agresivas en su- 
jetos comórbidos, como así también en aquellos únicamente depresivos y agresivos.

4.- Evaluar cuál es el papel del sexo dentro de los grupos con diferentes sintomatologías.

De acuerdo con la literatura revisada y los resultados previos, se esperó que:

1.- la depresión y la agresión física y verbal se asociaran significativamente,

2.- los sujetos comórbidos exhibieran una sintomatología clínica durante el período estudiado mostrando un mayor nivel de cronicidad,

3.- los sujetos comórbidos presentaran niveles significativamente superiores en las medidas de estudio que aquellos sujetos que sólo son depresivos o agresivos, como muestra de su mayor severidad y

4.- las respuestas sintomáticas comórbidas entre depresión y agresión fueran independientes del sexo.

\section{Metodología}

\section{MUESTRA}

Los resultados de esta investigación se obtuvieron a través de una muestra compuesta por 525 sujetos (58.9\% mujeres) que completaron los tres períodos de evaluación del que consta este estudio.

La selección de la muestra se realizó mediante un muestreo probabilístico aleatorio, tomando como parámetros poblacionales la edad y el sexo. Ambos parámetros se estimaron a partir del censo poblacional de la Comunidad Autónoma de Madrid. La media de edad en cada período de evaluación fue igual a 10.86 para el Tiempo 1 (T1), 11.86 para el Tiempo 2 (T2) y 12.86 para el Tiempo 3 (T3), con una desviación típica de 1.54 en cada período. La mayoría de las familias de procedencia de los sujetos evalua- dos tenía una estructura principalmente biparental $(92 \%)$ y se correspondía con un estrato económico medio (85\%), según el índice de Honllinsghead (1975).

Con la muestra seleccionada se constituyeron distintos grupos en función de la sintomatología presentada, en la que la pertenencia a un grupo era excluyente para pertenecer a otro. Los participantes fueron asignados a cada uno de los cinco grupos de estudio en función de su puntuación que, tomada en valores absolutos, se encontraba por encima de la media grupal más una desviación típica (Tabla 1 ).

Los grupos sintomáticos establecidos según la presencia o no de puntuaciones clínicamente significativas en las diferentes variables en el primer momento de medida, cuando los sujetos contaban con una media de edad de 11 años, fueron: comórbidos, es decir, integrados por aquellos sujetos que obtuvieron una puntación superior al punto de corte establecido en las medidas de depresión y agresión total (11 sujetos: 5 varones y 6 mujeres), depresivos (22 sujetos: 7 varones y 15 mujeres), agresivos físicos (3 sujetos: 3 varones), agresivos verbales (11 sujetos: 2 varones y 9 mujeres) y agresivos totales (20 sujetos: 9 varones y 11 mujeres).

\section{Procedimiento}

Se obtuvo de la Consejería de Madrid y de los centros educativos, la autorización para realizar la recolección de datos. Posteriormente y a través del tutor del aula, se solicitó la autorización de los padres mediante consentimiento informado sobre la participación de su hijo/a en la investigación.

Las pruebas fueron administradas por grupos de clase y en la recolección de datos colaboraron distintos psicólogos, previamente entrenados para llevar a cabo esta tarea. Antes del comienzo de la evaluación, se explicaban a los sujetos brevemente los objetivos y los instrumentos a administrar. Una vez dadas las instrucciones y resueltas las dudas de los alumnos, se procedió a la aplicación de los diferentes instrumentos, 
los cuales formaban parte de un amplio estudio longitudinal sobre la relación entre diferentes emociones infantiles.

La evaluación fue realizada por las mismas personas y en el mismo orden en las tres evaluaciones realizadas a lo largo de los tres cursos académicos.

\section{INSTRUMENTOS DE EVALUACIÓN}

\section{DEPRESIÓN}

Para medir el nivel de depresión en niños y adolescentes se utilizó la Primera Adaptación del CDI-S a Población Española (del Barrio, Roa, Olmedo \& Colodrón, 2002) del Children Depresión Inventory-Short-CDI-S(Kovacs, 1992). Este cuestionario es una versión de 10 ítemes cuya principal ventaja es su brevedad.

Los ítemes están enunciados en tres frases que representan tres niveles de intensidad de la sintomatología depresiva, a través de los cuales el sujeto debe elegir el ítem que indica el estado emocional experimentado en las dos últimas semanas. Esta prueba evalúa específicamente elementos relacionados con aspectos como la autoestima (por ej.: "nadie me quiere", "me odio", "soy feo" o "me siento solo siempre"), la anhedonia (por ej.: "tengo ganas de llorar todos los días", "estoy triste siempre" o "las cosas me preocupan siempre") y la desesperanza (por ej.: "nunca me saldrá nada bien", "todo lo hago mal" o "no tengo amigos"), excluyendo aspectos sintomáticos comunes a otros trastornos psicopatológicos infantiles, lo que lo convierte en un instrumento muy conveniente para evaluar la especificidad de la depresión infantil.

Esta versión abreviada tiene una consistencia interna adecuada obtenida mediante el alpha de Cronbach que oscila entre .71 y .74 en población normal española (del Barrio et al., 2002) y .79 en población americana (Kovacs, 1992). Por su parte, la validez concurrente con la prueba de la que tiene su origen alcanza el valor de $r=.89(p<.001)$ (del Barrio et al., 2002).
Estos datos confirman que a pesar de la disminución del número de ítemes, la prueba conserva las propiedades psicométricas del instrumento original. En la muestra de estudio la fiabilidad obtenida fue igual a .83 .

\section{AGRESIÓN FÍSICA Y VERBAL}

La agresión tanto física como verbal se evaluó con la versión española del Cuestionario de Agresividad Física y Verbal -AFV(del Barrio et al., 2001). Esta escala consta de 20 ítemes que recogen distintas conductas agresivas, tanto físicas (por ej.: "pego patadas y puñetazos" o "muerdo a otros") como verbales (por ej.: "insulto a los compañeros" o "amenazo a los otros"). El formato de respuesta es de tres alternativas (3: a menudo, 2: algunas veces y 3: nunca), según la frecuencia de aparición de la conducta. Su aplicación es individual o colectiva y está diseñada para sujetos de entre 7 y 17 años. Además, este cuestionario cuenta con unos ítemes control, ideados para evitar tendencias de respuesta. Con respecto a las características psicométricas de la prueba, la consistencia interna obtenida mediante el alpha de Cronbach oscila entre .73 y .86 (Caprara \& Pastorelli, 1993; del Barrio, Frías \& Mestre, 1994; del Barrio et al., 2001). La fiabilidad test-retest con un intervalo de 2 semanas en agresividad física y verbal es igual a $.70(p<.000)$, mientras que la validez de constructo con el Inventario de Depresión Infantil (CDI) fue igual a $.40(p<.001)$. Para la presente muestra de estudio la fiabilidad encontrada fue para la agresión física igual a .66 y para la verbal, .70 .

\section{ANÁLISIS DE DATOS}

En primer lugar se realizó un análisis descriptivo para poder estimar el punto de corte (media más una desviación típica). Estos descriptivos se presentan en la Tabla 1, agrupando la muestra por edad y sexo en cada uno de los tres momentos de evaluación. A continuación se exploró a través de 
un análisis de correlaciones de Pearson a dos colas, si existía una asociación significativa entre la depresión y la agresión en cada uno de los tipos medidos en este estudio (física, verbal y total). Por último, se llevó a cabo un análisis de variancia de diseño mixto con medidas repetidas en un solo factor (ANOVA-MR). El ANOVA-MR está especialmente indicado para muestras pequeñas ya que cada sujeto actúa como su propio control. Este hecho implica un análisis estadístico más complejo que requiere supuestos adicionales, tales como el de esfericidad. Dicho supuesto se cumple en todos los casos, menos en la evaluación de la depresión, para la cual se ha utilizado un análisis multivariado de la variancia (MANOVA). Por tanto, este tipo de análisis permite la evaluación longitudinal del cambio a lo largo del tiempo (Palmer, 2011; Ximénez \& San Martín, 2000). Asimismo, este análisis también permite comparar las puntuaciones obtenidas por los mismos sujetos en diferentes pruebas psicométricas, a fin de analizar la severidad de las respuestas depresiva y agresiva, a través de las diferencias de medias sobre los grupos estudiados a lo largo del tiempo.

Los análisis estadísticos se realizaron con el Programa Pasw Statistics, en su versión 18.0. Para el cálculo del tamaño del efecto se utilizó el Programa $G^{*}$ Power 3.1, desarrollado por Faul, Erdfelder, Lang y Buchner (2007).

\section{Resultados}

ANÁLISIS DE LAS RELACIONES ENTRE DEPRESIÓN Y AGRESIÓN ENTRE LOS DIFERENTES TIEMPOS DE MEDIDA

\section{ANÁLISIS DE CORRELACIONES}

Se encontraron asociaciones significativas entre la depresión y la agresión en los tres momentos de evaluación (ver Tabla 2), aunque el valor absoluto de la asociación variaba dependiendo del tipo de agresión evaluada. Así a los 11 y los 13 años, la asociación más potente se dio entre la depresión y la agresión verbal, mientras que a la edad de 12 años, fue la agresión total (física y verbal conjunta) la que estableció una mayor asociación con la depresión. Cabe destacar que a los 11 años, la depresión no se asoció significativamente con la agresión física, aunque sí con el resto de edades, obteniendo a los 12 años el mismo valor que la agresión verbal, y a los 13 años, el valor más bajo de los encontrados en los tres tipos de agresión explorados.

ANÁLISIS DE VARIANCIA CON DISEÑO MIXTO PARA MEDIDAS REPETIDAS EN UN SOLO FACTOR

ANÁLISIS DE LA CRONICIDAD DE LA AGRESIÓN Y LA DEPRESIÓN EN LOS DIFERENTES GRUPOS SINTOMÁTICOS

Como se representa en la Figura 1, los sujetos comórbidos mostraron puntuaciones clínicamente significativas desde los 11 hasta los 13 años en todos los tipos de agresión evaluados (verbal, física y total). Esto indica una cronicidad manifiesta en la respuesta agresiva. Con respecto a la depresión, se observa un patrón similar a los 11 y a los 12 años, pero no a los 13 años, edad en la que las puntuaciones clínicamente significativas descienden levemente por debajo del punto de corte establecido.

ANÁLISIS DE LA SEVERIDAD DE LA AGRESIÓN Y LA DEPRESIÓN EN LOS DIFERENTES GRUPOS SINTOMÁTICOS

Los resultados mostraron que existen diferencias significativas entre los distintos grupos sintomáticos medidos (ver Tabla 3), lo que indica distintos grados de severidad exhibida a lo largo del período de estudio. En la variable dependiente agresión verbal, los análisis señalan que ésta es significativamente más alta $[F(4,58)=10.485, p=.000$, $\left.\eta_{\mathrm{p}}{ }^{2}=.42\right]$, en los sujetos comórbidos, agresivos verbales y totales, respecto de los sujetos depresivos.

Al analizar la respuesta en agresión física, se encuentra que los niveles de este tipo de 
agresión fueron significativamente más elevados $[F(4,58)=11.084, p=.000$, $\left.\eta_{\mathrm{p}}{ }^{2}=.43\right]$, en el grupo comórbido y agresivo total, frente a los grupos depresivos y agresivos verbales. Tomando la agresión total como variable dependiente, los resultados indican que los grupos comórbidos y agresivos totales muestran los niveles más altos de agresión total, difiriendo ambos significativamente $\left[F(4,58)=12.403, p=.000, \eta_{\mathrm{p}}{ }^{2}=.46\right]$, con el grupo depresivo, mientras que de forma única se establece una diferencia significativa entre el grupo comórbido y el grupo de agresión verbal. Por último, en el análisis de la respuesta depresiva, los resultados indican que los grupos comórbido y depresivo muestran la mayor severidad, y que ambos muestran diferencias significativas sobre el resto de sujetos $[F(4,58)=27.717, p=.000$, $\left.\eta_{\mathrm{p}}{ }^{2}=.66\right]$, caracterizados por su sintomatología agresiva de tipo física, verbal y total.

Cabe destacar que los resultados no indicaron diferencia significativa alguna por sexo en las respuestas depresivas y agresivas medidas, ni cuando se midió de forma única, ni cuando se hizo en interacción con los distintos grupos sintomáticos (ver Tabla 3).

\section{Discusión}

El presente artículo pretende analizar longitudinalmente la comorbilidad entre agresión y depresión, tanto desde una perspectiva estadística como clínica, es decir, no sólo en términos de covariación sino también en términos de severidad y cronicidad. La investigación previa sobre este tema ha infravalorado a veces el estudio de la comorbilidad, analizando muestras en las que la aparición conjunta de la depresión y la agresión estaba poco representada (Weersing \& Weisz, 2002), o analizando la conducta agresiva como un desorden de conducta antisocial global (Capaldi, 1992; Ezpeleta et al., 2005, 2006; Fergusson, Lynskey \& Horwood, 1996; Frías et al., 2009), o como parte de una alteración de amplia banda (interiorizado vs. exteriorizado) de las que la depresión y agresión forman parte (Evans \&
Frank, 2004; Gjone \& Stevenson, 1997; Lilienfeld, 2003; Marmorstein, 2007). Los resultados obtenidos en este trabajo, no sólo evalúan específicamente los niveles de depresión y agresión como alteraciones independientes en grupos con diferentes tipos de comorbilidad, sino que también analizan las relaciones entre ellos a lo largo del tiempo. Esto ha permitido superar las limitaciones inherentes a los diseños transversales en el estudio de la comorbilidad, así como las limitaciones propias de los sesgos del recuerdo propio de los análisis retrospectivos (Angold et al., 1999). Asimismo, tal y como estos autores sugieren, el análisis de la comorbilidad sobre una muestra procedente de la población general es lo más idóneo, en la medida que evita los sesgos de severidad y problemas propios de las muestras clínicas.

Los presentes resultados mostraron que la variación en los niveles de depresión inciden sobre la variación de los niveles de agresión $\mathrm{y}$ viceversa, y que esta reciprocidad ocurre con una determinada potencia y dirección. Por tanto, más allá de la mera co-ocurrencia, existe una asociación significativa entre estas dos alteraciones, lo que está en sintonía con una de las dos premisas establecidas por Clark y Watson (1991), la estadística. En concreto, los resultados hallados indican una relación positiva moderada entre depresión y agresión que ha oscilado entre .11 y .24 en función de la media de edad de los sujetos y del tipo de agresión. Dicha asociación, aunque significativa, se encuentra por debajo del rango observado en estudios previos, la cual en función de los diferentes trabajos ha oscilado entre .34 y .67 (del Barrio et al., 2001; Garber et al., 1991; Messer \& Gross, 1994; Morrow, Hubbar, Rubin \& Mc Auliffe, 2008; Warrick-Swansen, 1999; Weiss \& Catron, 1994). Cabe destacar que las correlaciones encontradas en los presentes datos fueron más potentes con la agresión verbal en todas las edades que con la física, excepto a los 12 años, en que se mostraron equiparables. Estos resultados sugieren la conveniencia de explorar la comorbilidad con diferentes tipos de agresión $\mathrm{y}$ en diferentes grupos de edad. Aquellos 
trabajos que no consideran la agresión verbal, están prescindiendo de un porcentaje importante de la variabilidad compartida entre la agresión y la respuesta depresiva.

Las diferencias halladas en los diferentes tiempos estudiados, pone de manifiesto el carácter posiblemente evolutivo que tienen estas relaciones y la importancia de analizarlas tanto en función de la edad de los sujetos como del tipo de manifestación agresiva, física o verbal. Consistentemente con esta afirmación, el aumento de la asociación entre depresión y agresión con la edad también sugiere la importancia de la detección precoz.

Contrastada la relación entre agresión y depresión, se analizó la prevalencia de la comorbilidad depresivo-agresiva. Los resultados mostraron que la prevalencia de la comorbilidad encontrada en la población infanto-juvenil española es, en general, muy similar a la hallada en los estudios encontrados en la literatura (Messer \& Gross, 1994; Quiggle et al., 1992; Rudolph \& Clark, 2001). En concreto, la prevalencia total hallada fue de $10.1 \%$, en el rango de edad de 9 a 15 años. Aunque no aparecieron diferencias por sexo, la prevalencia encontrada en mujeres $(10.7 \%)$ fue más elevada que la encontrada en varones $(9.3 \%)$. Estos resultados muestran que los niveles de comorbilidad permanecen similares a los documentados en los últimos 15 años, lo que sugiere la necesidad de diseñar nuevas actuaciones preventivas para disminuirla e incidir sobre sus consecuencias (Bennett, 1994; Beyers \& Loeber, 2003; Chorpita \& Daleiden, 2002; Ezpeleta et al., 2006; Teesson et al., 2005; Youngstrom et al., 2003).

Como han informado diferentes trabajos, la comorbilidad a menudo se asocia con mayores daños, peores respuestas al tratamiento y un peor curso en las conductas problemas (Beyers \& Loeber, 2003; Clark \& Watson, 1991; Clark et al., 1995; Teesson et al., 2005; Youngstrom et al., 2003). Es por ello que nuestro interés se centró también en estudiar la cronicidad y severidad asociada a la comorbilidad entre depresión y agresión a lo largo del tiempo. Esto nos permitiría con- trastar la segunda premisa del modelo de Clark y Watson relativa a la sinergia esperable entre dos trastornos comórbidos. Los resultados contrastaron parcialmente la hipótesis previa, ya que el grupo comórbido es el único que mantiene sus puntuaciones por encima del punto de corte en los diferentes tipos de sintomatología a lo largo del período estudiado (de los 11 a los 13 años), excepto en depresión en el tercer momento de medida. Este patrón de mayor cronicidad es coincidente con la literatura previa (Angold \& Costello, 1992). Además, el patrón mostrado por los sujetos comórbidos, muy similar al del grupo de sujetos agresivos de tipo total en todas las medidas agresivas evaluadas (física, verbal y total), así como al grupo depresivo en medidas de depresión, está en consonancia con los estudios que han indicado que los sujetos comórbidos expresan las características de los grupos de los que están compuestos, exacerbando los síntomas de ambos. Este efecto se ha explicado por un aumento de la afectividad negativa subyacente o de los procesos cognitivos compartidos por ambas alteraciones, que elevan la probabilidad de una atribución hostil de la intención o la indefensión (Quiggle et al., 1992; Rudolph \& Clark, 2001), así como de la expresión de la ira (Alcázar-Córcoles, Verdejo-García, Bouso-Saiz \& Bezos-Saldaña, 2010; Kashani, Suarez, Allan \& Reid, 1997; Kerr \& Schneider, 2008).

En definitiva, los resultados apoyan aquellos que han encontrado que los grupos comórbidos exhiben una mayor cronicidad de la sintomatología evaluada (depresión, agresión física, agresión verbal y agresión total). En este sentido, se puede afirmar que la comorbilidad entre depresión y agresión, como se señaló al principio, se asocia con un peor curso de la sintomatología, la cual puede también afectar a la respuesta al tratamiento. Por tanto, es recomendable que en aquellos sujetos con una alteración depresiva o agresiva de larga duración se exploren conductas pasadas de aquella afectación que pudieran estar enmascaradas por los síntomas actuales y a los que pudiera subyacer una patología crónica. 
Con respecto a la severidad de los síntomas, se confirma parcialmente la hipótesis planteada, pues el grupo comórbido obtuvo las medias más altas en todas las respuestas evaluadas, excepto en agresión física en la que comórbidos y agresivos totales exhiben medias similares. Estos resultados ponen de relieve la posible existencia de factores comunes subyacentes a ambos trastornos que pudieran explicar la similaridad exhibida entre sujetos comórbidos y sujetos depresivos, por una parte, y sujetos agresivos, por otra (Ezpeleta et al., 2005; Hale et al., 2005; Rowe et al., 2008; Weiss, Süsser \& Catron, 1998; Wolff \& Ollendick, 2006).

Como se desprende de los resultados hallados, existe un elevado porcentaje de sujetos comórbidos que padecen una constelación de síntomas depresivos y agresivos. La identificación de un adolescente como agresivo o depresivo sin la adecuada exploración de la sintomatología comórbida asociada puede hacer ineficaz el tratamiento, como así también facilitar la recaída. De todo ello, se evidencia la necesidad de una detección correcta de los individuos comórbidos que disminuya el riesgo de su cronicidad y severidad. En cuanto al sexo, los datos hallados en este estudio indicaron la ausencia de un efecto significativo en el grupo comórbido, confirmando de nuevo nuestra hipótesis previa. Estos resultados son consistentes con los hallados por Knox y colaboradores (2000) en población clínica, revelando que una patología comórbida como la depresiva-agresiva en niños y adolescentes de población normal, hace desaparecer las diferencias esperables que el sexo modula en la expresión de la agresión y la depresión (Bruce et al., 2006; Canals, Doménech-Llaberia, Fernández-Ballart \& Martí-Henneberg, 2002; Keenan \& Hipwell, 2005; Knox et al., 2000; Ortega \& Monks, 2005).
A pesar de las ventajas metodológicas del estudio realizado, el trabajo que se informa no está exento de limitaciones. Cabe mencionar en primer lugar, la ausencia de padres y maestros como informantes, aunque distintos autores han señalado que los autoinformes sobre la conducta agresiva (Caprara \& Pastorelli, 1993; Knox et al., 2000), así como depresiva (Achenbach, 2008; Achenbach, Magrab \& Wohlford, 1990; Achenbach \& Maruish, 1999) ofrece totales garantías psicométricas para establecer inferencias a través de la información proporcionada. Igualmente, los análisis realizados se han obtenido sobre medidas dimensionales de la sintomatología depresiva y agresiva, por lo que tales resultados pudieran no ser totalmente coincidentes cuando dichas medidas se obtengan categóricamente. Por último, se indica que el grupo con puntuaciones clínicamente significativas en agresión física, sólo estaba formado por varones, por lo que las conclusiones respecto al sexo en lo que se refiere a este grupo quedan restringidas y han de considerarse con cautela.

Líneas futuras de trabajo deberán considerar estas limitaciones con el fin de superarlas. Futuros estudios deberán explorar el comportamiento de la agresión verbal en relación con otro tipo de alteraciones. Sería conveniente que posteriores investigaciones incrementaran los períodos de estudio desde edades más precoces hasta la edad adulta, como así también explorar estas relaciones con diferentes dimensiones del constructo depresión (anhedonia, disforia, baja autoestima). El análisis de la fuerza de los distintos factores de riesgo comunes que subyacen a la depresión y agresión y su incidencia en función del sexo y la edad quedan como tareas futuras pendientes. 


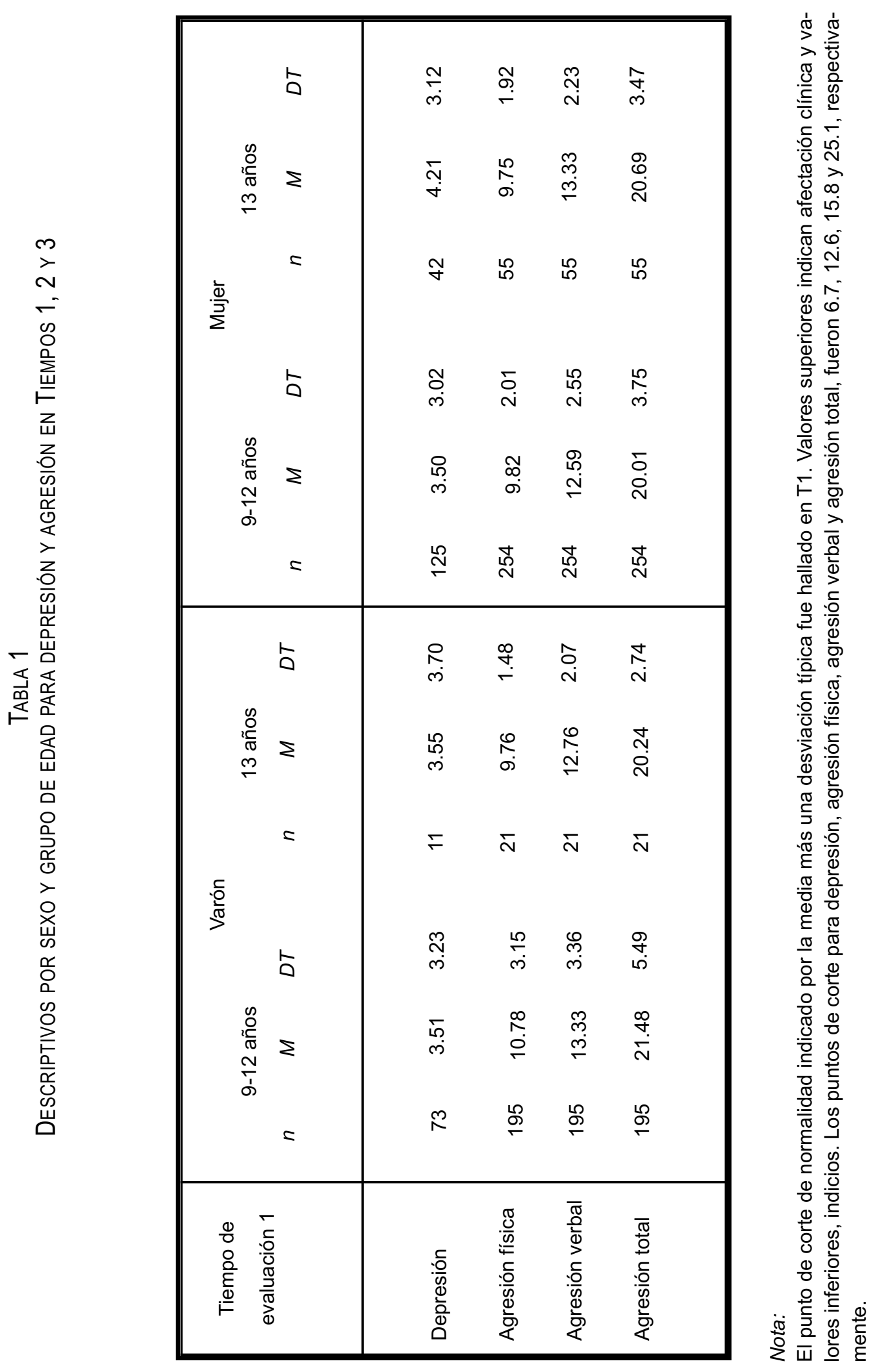


Gordillo, del Barrio y Carrasco

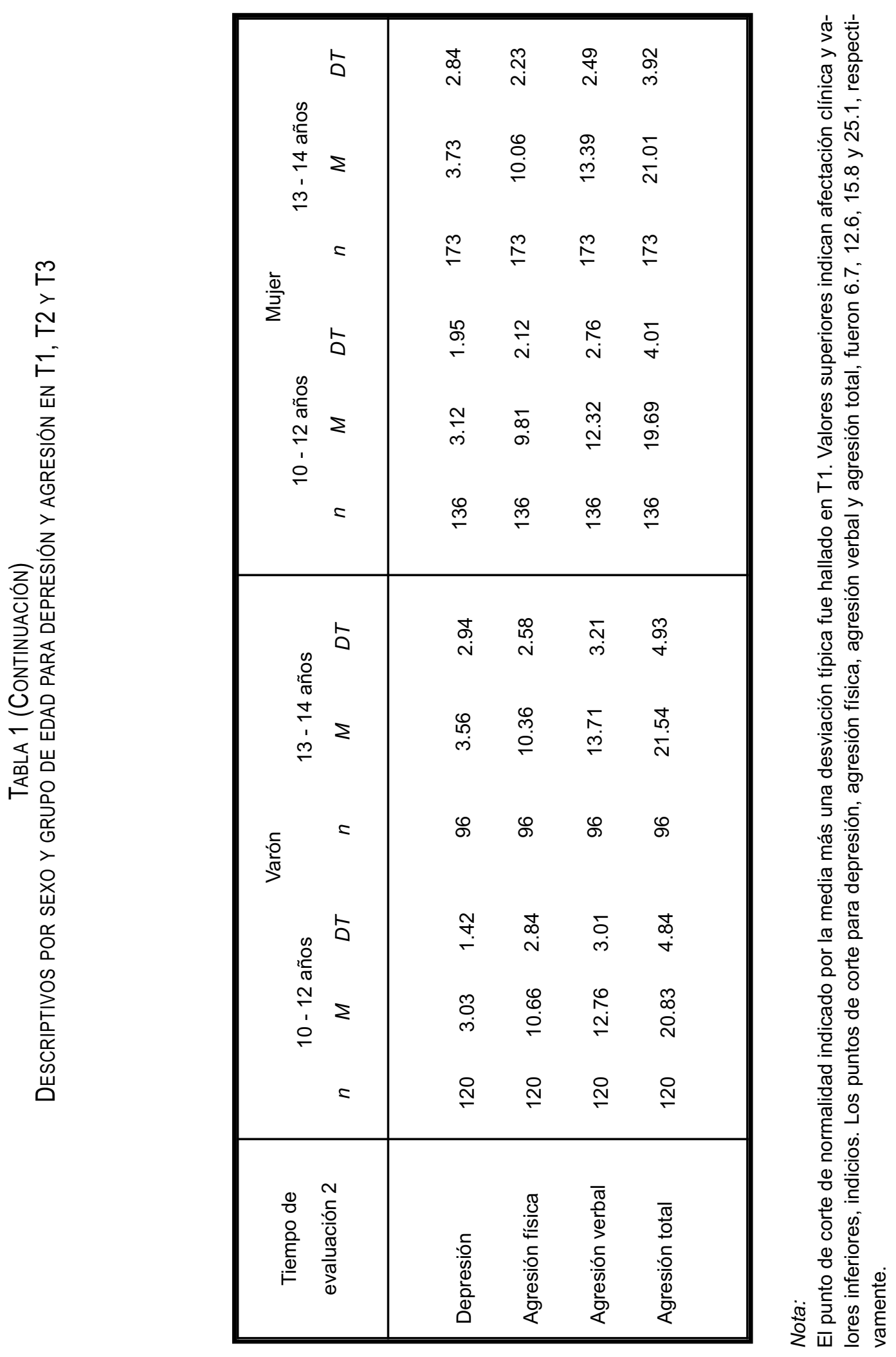

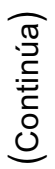




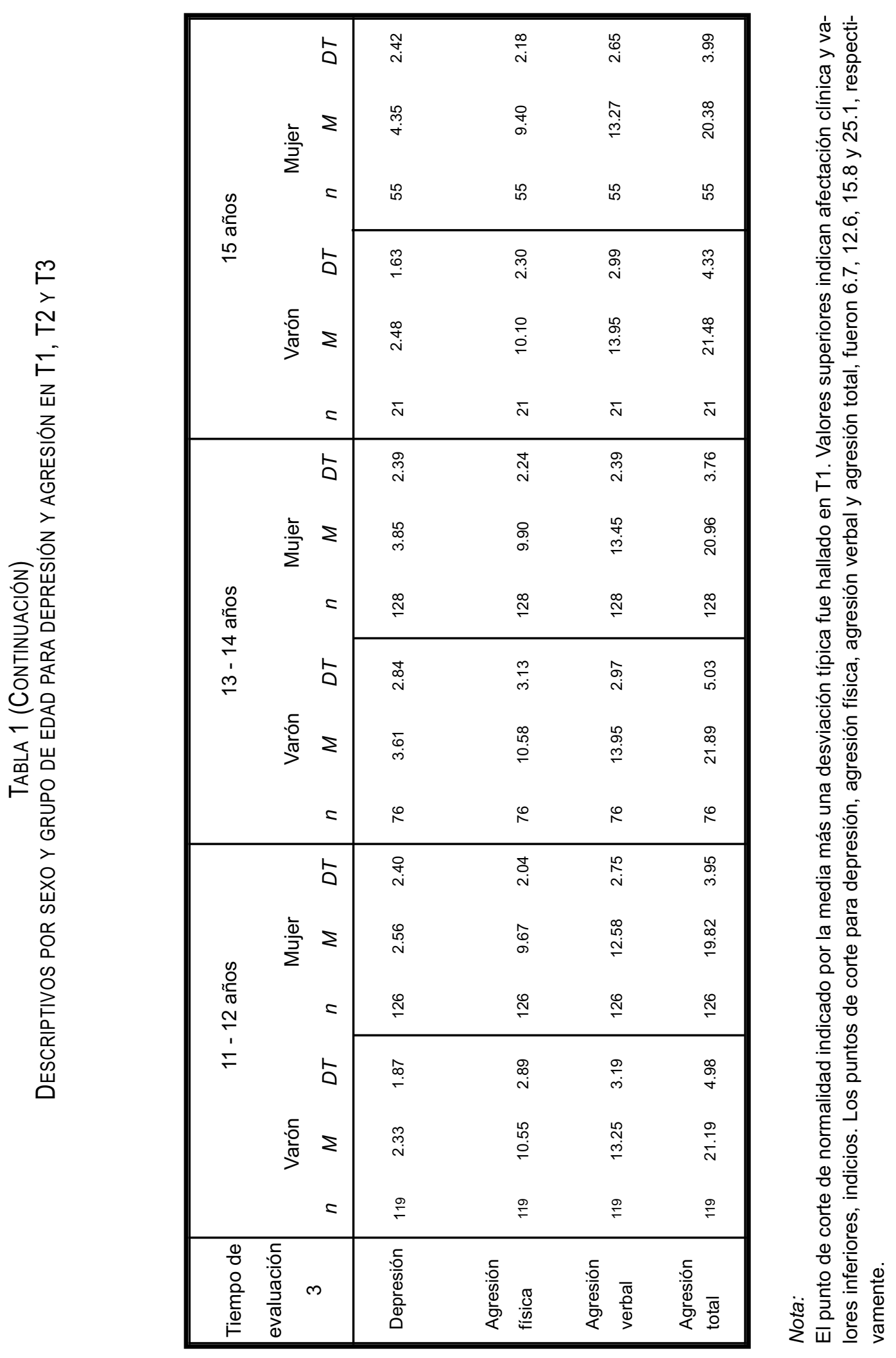


Gordillo, del Barrio y Carrasco

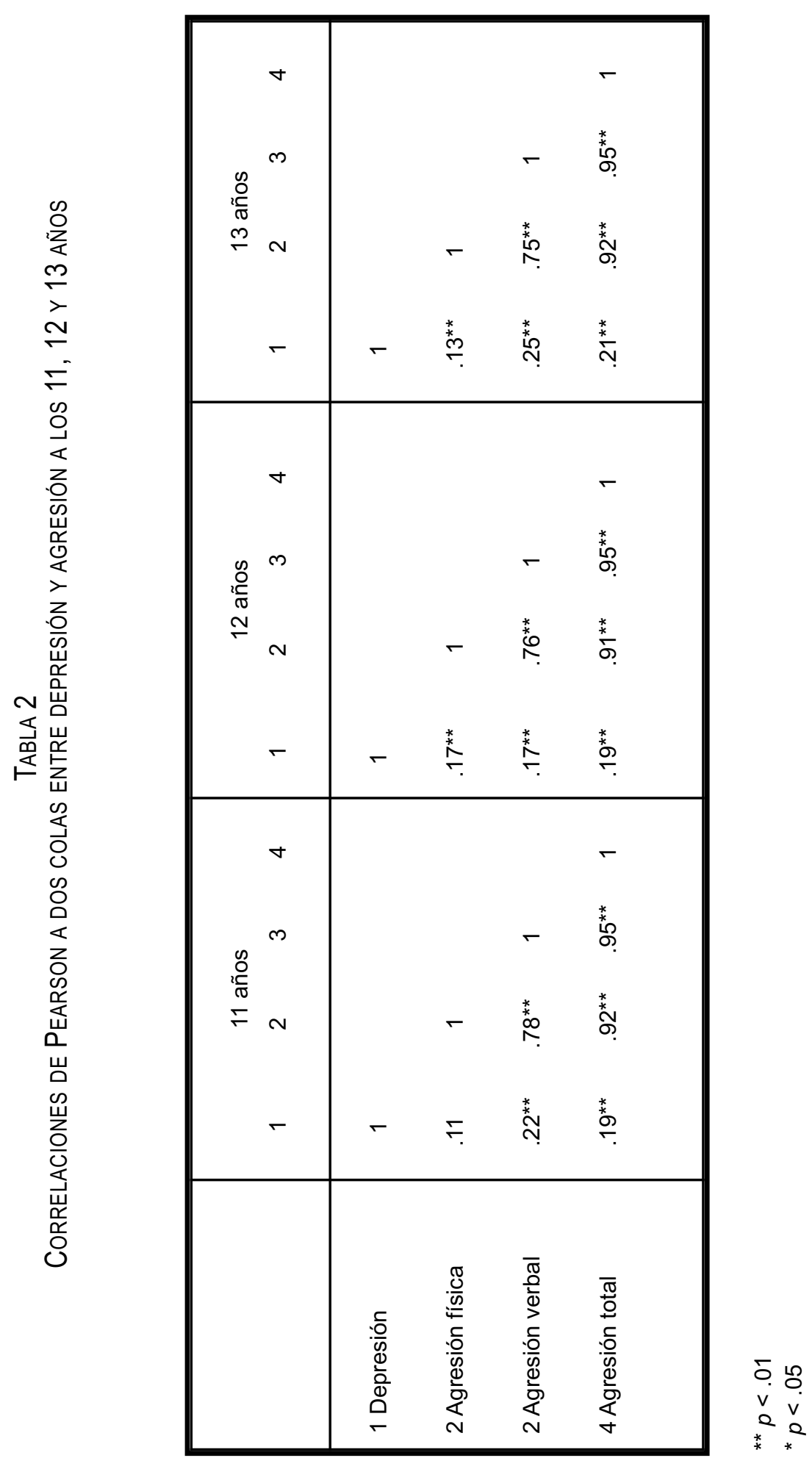


Comorbilidad entre depresión y agresión

TABLA 3

MEDIAS MARGINALES ESTIMADAS Y EFECTOS INTERSUJETOS SEGÚN EL GRUPO SINTOMÁTICO DE ESTUDIO DESDE LOS 11 A LOS 13 AÑOS

\begin{tabular}{|c|c|c|c|c|c|c|}
\hline Grupo Sintomático & $M$ & $D T$ & Anova intersujetos & $F$ & $p$ & $\eta 2$ \\
\hline $\begin{array}{l}\text { Comórbidos } \\
\text { Depresivos } \\
\text { Agresivos físicos } \\
\text { Agresivos verbales } \\
\text { Agresivos totales }\end{array}$ & \begin{tabular}{|c} 
Agresión verbal \\
16.689 \\
13.357 \\
$15.000^{\mathrm{a}}$ \\
14.991 \\
15.847
\end{tabular} & $\begin{array}{l}.460 \\
.348 \\
.878 \\
.594 \\
.342\end{array}$ & $\begin{array}{l}\text { Grupo sintomático } \\
\text { Sexo }^{\mathrm{c}} \\
\text { Grupo sintomático } \times \text { Sexo }^{d}\end{array}$ & $\begin{array}{c}10.485 \\
.692 \\
.567\end{array}$ & $\begin{array}{l}.000 \\
.409 \\
.639\end{array}$ & $\begin{array}{l}.420 \\
.012 \\
.028\end{array}$ \\
\hline $\begin{array}{l}\text { Comórbidos } \\
\text { Depresivos } \\
\text { Agresivos físicos } \\
\text { Agresivos verbales } \\
\text { Agresivos totales }\end{array}$ & \begin{tabular}{|c} 
Agresión física \\
13.106 \\
10.068 \\
$12.889^{\mathrm{a}}$ \\
10.583 \\
13.195
\end{tabular} & $\begin{array}{l}.508 \\
.384 \\
.968 \\
.655 \\
.377\end{array}$ & $\begin{array}{l}\text { Grupo sintomático } \\
\text { Sexo }^{\mathrm{c}} \\
\text { Grupo sintomático } \times \text { Sexo }^{d}\end{array}$ & $\begin{array}{c}11.084 \\
1.849 \\
.323\end{array}$ & $\begin{array}{l}.000 \\
.179 \\
.809\end{array}$ & $\begin{array}{l}.433 \\
.031 \\
.016\end{array}$ \\
\hline $\begin{array}{l}\text { Comórbidos } \\
\text { Depresivos } \\
\text { Agresivos físicos } \\
\text { Agresivos verbales } \\
\text { Agresivos totales }\end{array}$ & \begin{tabular}{|c|} 
Agresión total \\
26.606 \\
20.973 \\
$24.778^{\mathrm{a}}$ \\
22.907 \\
25.919
\end{tabular} & $\begin{array}{l}.780 \\
.590 \\
1.488 \\
1.008 \\
.579\end{array}$ & 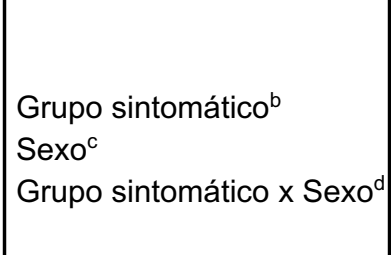 & $\begin{array}{c}12.403 \\
1.451 \\
.313\end{array}$ & $\begin{array}{l}.000 \\
.233 \\
.816\end{array}$ & $\begin{array}{l}.461 \\
.024 \\
.016\end{array}$ \\
\hline $\begin{array}{l}\text { Comórbidos } \\
\text { Depresivos } \\
\text { Agresivos físicos } \\
\text { Agresivos verbales } \\
\text { Agresivos totales }\end{array}$ & $\begin{array}{c}\text { Depresión } \\
8.378 \\
7.837 \\
2.778^{\mathrm{a}} \\
3.074 \\
2.591\end{array}$ & $\begin{array}{c}.594 \\
.449 \\
1.133 \\
.767 \\
.441\end{array}$ & $\begin{array}{l}\text { Grupo sintomático } \\
\text { Sexo }^{\mathrm{c}} \\
\text { Grupo sintomático } \times \text { Sexo }^{d}\end{array}$ & $\begin{array}{c}27.717 \\
.420 \\
2.474\end{array}$ & $\begin{array}{l}.000 \\
.519 \\
.070\end{array}$ & $\begin{array}{l}.657 \\
.007 \\
.113\end{array}$ \\
\hline
\end{tabular}

Nota:

a basado en la media marginal poblacional modificada

${ }^{\mathrm{b}} \mathrm{gl}=4,58$

${ }^{\mathrm{c}} \mathrm{gl}=1,58$

${ }^{\mathrm{d}} g l=3,58$ 
FIGURA 1

TENDENCIAS LONGITUDINALES DE LOS SUJETOS COMÓRBIDOS EN COMPARACIÓN CON EL RESTO DE GRUPOS SINTOMÁTICOS SOBRE LAS DIFERENTES RESPUESTAS DEPRESIVAS Y AGRESIVAS

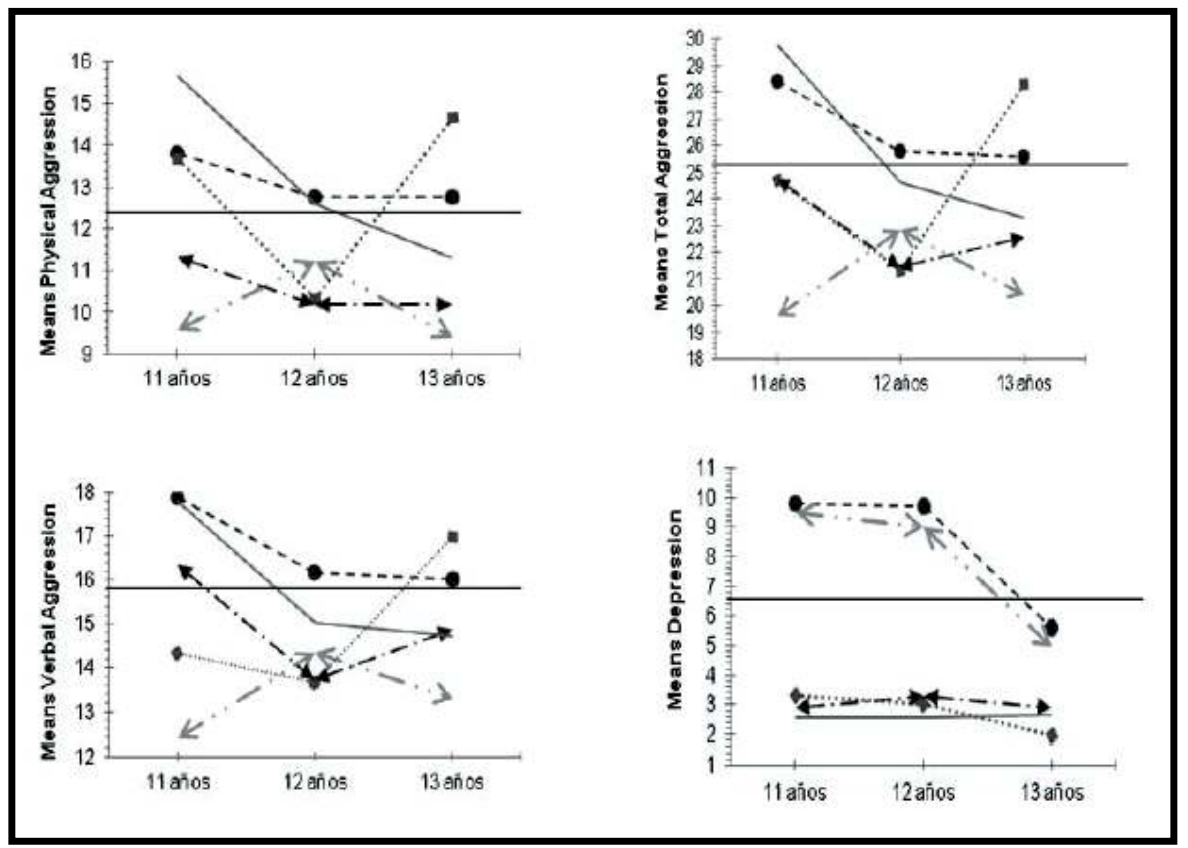

\section{Notación:}

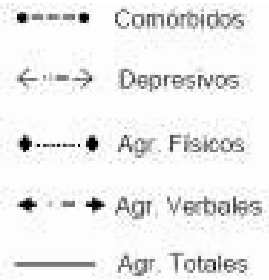

La línea marca la puntuación clínicamente significativa 


\section{REFERENCIAS BIBLIOGRÁFICAS}

Achenbach, T.M. (2008). Assessment, diagnosis, nosology, and taxonomy of child and adolescent psychopathology. En M. Hersen \& A.M. Gross (Eds.), Handbook of clinical psychology: Vol. 2. Children and adolescents (pp. 429-457). Hoboken, NJ, US: Wiley.

Achenbach, T.M., Magrab, P.R. \& Wohlford, P. (1990). Developmental psychopathology as a conceptual framework for training in multiple settings. En P.R. Magrab \& P. Wohlford (Eds.), Improving psychological services for children and adolescents with severe mental disorders: Clinical training in psychology (pp. 79-84). Washington, DC, US: American Psychological Association.

Achenbach, T.M. \& Maruish, M.E. (1999). The child behavior checklist and related instruments. En M.E. Maruish (Ed.), The use of psychological testing for treatment planning and outcomes assessment (2nd ed., pp. 429-466). Mahwah, NJ, US: Erlbaum.

Alcázar-Córcoles, M.A., Verdejo-García, A., Bouso-Saiz, J.C. \& Bezos-Saldaña, L. (2010). Neuropsicología de la agresión impulsiva. Revista de Neurología, 50(05), 291-299.

Angold, A. \& Costello, E.J. (1992). Comorbidity in children and adolescents with depression. Child and Adolescent Psychiatric Clinics of North America, 1(1), 31-51.

Angold, A. \& Costello, E.J. (1993). Depressive comorbidity in children and adolescents: Empirical, theoretical, and methodological issues. American Journal of Psychiatry, 150 (12), 1779-1791.

Angold, A., Costello, E.J. \& Erkanli, A. (1999). Comorbidity. Journal of Child Psychology and Psychiatry, 40(1), 57-87. doi: 10.1111/ 1469-7610.00424.

Bennett, D.S. (1994). Depression among children with chronic medical problems: A meta- analysis. Journal of Pediatric Psychology, 19 (2), 149-169. doi: 10.1093/jpepsy/19.2.149.

Beyers, J.M. \& Loeber, R. (2003). Untangling developmental relations between depressed mood and delinquency in male adolescents. Journal of Abnormal Child Psychology, 31(3), 247-266. doi: 10.1023/a:1023225428957.

Bruce, A.E., Cole, D.A., Dallaire, D.H., Jacquez, F.M., Pineda, A.Q. \& LaGrange, B. (2006). Relations of parenting and negative life events to cognitive diatheses for depression in children. Journal of Abnormal Child Psychology, 34(3), 321-333.

Canals, J., Doménech-Llaberia, E., FernándezBallart, J. \& Martí-Henneberg, C. (2002). Predictors of depression at eighteen. A 7-year follow-up study in a Spanish nonclinical population. European Child \& Adolescent Psychiatry, 11(5), 226-233.

Capaldi, D.M. (1992). Co-occurrence of conduct problems and depressive symptoms in early adolescent boys: II. A 2-year follow-up at grade 8. Development and Psychopathology, 4(1), 125-144.

Caprara, G.V. \& Pastorelli, C. (1993). Early emotional instability, prosocial behaviour, and aggression: Some methodological aspects. European Journal of Personality, 7(1), 19-36. doi: 10.1002/per.2410070103.

Clark, L.A. \& Watson, D. (1991). Tripartite model of anxiety and depression: Psychometric evidence and taxonomic implications. Journal of Abnormal Psychology, 100(3), 316-336. doi: 10.1037/0021-843X.100.3.316.

Clark, L.A., Watson, D. \& Reynolds, S. (1995). Diagnosis and classification of psychopathology: Challenges to the current system and future directions. Annual Review of Psychology, 46, 121-153.

Crockett, L.J. \& Randall, B.A. (2006). Linking adolescent family and peer relationships to the quality of young adult romantic relationships: The mediating role of conflict tactics. Journal 
of Social and Personal Relationships, 23(5), 761-780.

Cyranowski, J.M., Frank, E., Young, E. \& Shear, K. (2000). Adolescent onset of the gender difference in lifetime rates of major depression. Archives of General Psychiatry, 57(1), 21-27.

Chorpita, B.F. (2002). The tripartite model and dimensions of anxiety and depression: An examination of structure in a large school sample. Journal of Abnormal Child Psychology, 30(2), 177-190.

Chorpita, B.F. \& Daleiden, E.L. (2002). Tripartite dimensions of emotion in a child clinical sample: Measurement strategies and implications for clinical utility. Journal of Consulting and Clinical Psychology, 70(5), 1150-1160.

de Beurs, E., den Hollander-Gijsman, M.E., Helmich, S. \& Zitman, F.G. (2007). The tripartite model for assessing symptoms of anxiety and depression: Psychometrics of the Dutch version of the mood and anxiety symptoms questionnaire. Behaviour Research and Therapy, 45(7), 1609-1617. doi: 10.1016/ j.brat.2006.07.004.

del Barrio, V., Frías, D. \& Mestre, V. (1994). Autoestima y depresión en niños [Self-esteem and depression in children]. Revista de Psicología General y Aplicada, 47(4), 471-476.

del Barrio, V., Moreno, C. \& López, R. (2001). Evaluación de la agresión y la inestabilidad emocional en niños españoles: Su relación con la depresión [Assessment of aggression and emotional instability in Spanish children: its relationship with depression]. Clinica y $\mathrm{Sa}$ lud, 12(1), 33-50.

del Barrio, V., Roa, L., Olmedo, M. \& Colodrón, F. (2002). Primera adaptación del CDI-S a población española [First adaptation of the CDI$\mathrm{S}$ for Spanish population]. Acción Psicológica, 3, 263-274.

Evans, A.S. \& Frank, S.J. (2004). Adolescent depression and externalizing problems: Test- ing two models of comorbidity in an inpatient sample. Adolescence, 39(153), 1-18.

Ezpeleta, L., Domenech, J.M. \& Angold, A. (2006). A comparison of pure and comorbid CD/ODD and depression. Journal of Child Psychology and Psychiatry, 47(7), 704-712. doi: 10.1111/j.1469-7610.2005.01558.x.

Ezpeleta, L., Granero, R. \& Domenech, J.M. (2005). Differential contextual factors of comorbid conduct and depressive disorders in Spanish children. European Child \& Adolescent Psychiatry, 14(5), 282-291. doi: 10. 1007/s00787-005-0476-5.

Faul, F., Erdfelder, E., Lang, A.G. \& Buchner, A. (2007). G*Power 3: A flexible statistical power analysis program for the social, behavioral, and biomedical sciences. Behavior Research Methods, 39(2), 175-191.

Fergusson, D.M., Lynskey, M.T. \& Horwood, L.J. (1996). Origins of comorbidity between conduct and affective disorders. Journal of the American Academy of Child \& Adolescent Psychiatry, 35(4), 451-460. doi: 10.1097/00 004583-199604000-00011.

Frías, A., Carrasco, A., Fernández, M.J., García, A. \& García, B. (2009). Comorbilidad entre trastornos de conducta y depresión mayor en la adolescencia: Prevalencia, teorías explicativas y estatus nosológico [Comorbidity between conduct disorder and major depression in adolescence: Prevalence, explanatory theories, and nosological status]. Revista de Psicopatología y Psicología Clínica, 14(1), 1-16.

Garber, J., Quiggle, N.L., Panak, W., Dodge, K.A., Cicchetti, D. \& Toth, S.L. (1991). Aggression and depression in children: Comorbidity, specificity, and social cognitive processing. En D. Chicchetti \& L. Sheree (Eds.), Internalizing and externalizing expressions of dysfunction (Vol. 2, pp. 225-264). Hillsdale, NJ, England: Erlbaum.

Gjone, H. \& Stevenson, J. (1997). A longitudinal twin study of temperament and behavior 
problems: Common genetic or environmental influences? Journal of the American Academy of Child \& Adolescent Psychiatry, 36(10), 1448-1456.

Hale, W.W. III., Van Der Valk, I., Engels, R. \& Meeus, W. (2005). Does perceived parental rejection make adolescents sad and mad? The association of perceived parental rejection with adolescent depression and aggression. Journal of Adolescent Health, 36(6), 466-474. doi: 10.1016/j.jadohealth.2004.04.007.

Hammen, C. \& Compas, B.E. (1994). Unmasking unmasked depression in children and adolescents: The problem of comorbidity. Clinical Psychology Review, 14(6), 585-603. doi: 10.1016/0272-7358(94)90018-3.

Harter, S. (1998). The effects of child abuse on the self-system. Journal of Aggression, Maltreatment \& Trauma, 2(1), 147-169.

Harter, S. \& Monsour, A. (1992). Development analysis of conflict caused by opposing attributes in the adolescent self-portrait. Developmental Psychology, 28(2), 251-260.

Higa-McMillan, C.K., Smith, R.L., Chorpita, B.F. \& Hayashi, K. (2008). Common and unique factors associated with DSM-IV-TR internalizing disorders in children. Journal of Abnormal Child Psychology, 36(8), 12791288. doi: 10.1007/s10802-008-9250-8.

Hogansen, J.M. (2004). Trajectories of co-occurring aggressive and depressive symptoms in children: Prediction from child and family characteristics. Dissertation Abstracts International: Section B: The Sciences and Engineering, 65(6-B), 3163.

Jensen, P.S. (2003). Comorbidity and child psychopathology: Recommendations for the next decade. Journal of Abnormal Child Psychology, 31(3), 293-300. doi: 10.1023/a:1023281 513936.

Kashani, J.H., Suarez, L., Allan, W.D. \& Reid, J.C. (1997). Hopelessness in inpatient youths: A closer look at behavior, emotional expres- sion, and social support. Journal of the American Academy of Child \& Adolescent Psychiatry, 36(11), 1625-1631. doi: 10.1097/ 00004583-199711000-00028.

Kazdin, A.E., French, N.H., Unis, A.S. \& EsveldtDawson, K. (1983). Assessment of childhood depression: Correspondence of child and parent ratings. Journal of the American Academy of Child Psychiatry, 22(2), 157- 164.

Keenan, K. \& Hipwell, A.E. (2005). Preadolescent clues to understanding depression in girls. Clinical Child \& Family Psychology Review, 8(2), 89-105.

Keiley, M.K., Lofthouse, N., Bates, J.E., Dodge, K.A. \& Pettit, G.S. (2003). Differential risks of covarying and pure components in mother and teacher reports of externalizing and internalizing behavior across ages 5 to 14 . Journal of Abnormal Child Psychology, 31(3), 267-283. doi: 10.1023/A:1023277413027.

Kerr, M.A. \& Schneider, B.H. (2008). Anger expression in children and adolescents: A review of the empirical literature. Clinical Psychology Review, 28(4), 559-577. doi: 10.101 6/j.cpr.2007.08.001.

Knox, M., King, C., Hanna, G.L., Logan, D. \& Ghaziuddin, N. (2000). Aggressive behavior in clinically depressed adolescents. Journal of the American Academy of Child \& Adolescent Psychiatry, 39(5), 611-618. doi: 10.1097/000 04583-200005000-00015.

Kovacs, M. (1992). Children's Depression Inventory, CDI. Toronto: Multi-Heath Systems Inc. Lambert, S.F., McCreary, B.T., Joiner, T.E., Schmidt, N.B. \& Ialongo, N.S. (2004). Structure of anxiety and depression in urban youth: An examination of the tripartite model. Journal of Consulting and Clinical Psychology, 72 (5), 904-908.

Lefkowitz, M.M. \& Tesiny, E.P. (1985). Depression in children: Prevalence and correlates. Journal of Consulting and Clinical Psychology, 53(5), 647-656. 
Lewinsohn, P.M., Rohde, P., Seeley, J.R. \& Hops, H. (1991). Comorbidity of unipolar depression: I. Major depression with dysthymia. Journal of Abnormal Psychology, 100(2), 205-213. doi: 10.1037/0021-843x. 100.2.205.

Lilienfeld, S.O. (2003). Comorbidity between and within childhood externalizing and internalizing disorders: Reflections and directions. Journal of Abnormal Child Psychology, 31(3), 285-291. doi: 10.1023/a: 1023 229529866.

Marmorstein, N.R. (2007). Relationships between anxiety and externalizing disorders in youth: The influences of age and gender. Journal of Anxiety Disorders, 21(3), 420-432. doi: 10. 1016/j.janxdis.2006.06.004.

Messer, S.C. \& Gross, A.M. (1994). Childhood depression and aggression: A covariance structure analysis. Behaviour Research and Therapy, 32(6), 663-677. doi: 10.1016/00057967(94)90023-X.

Morrow, M.T., Hubbard, J.A., Rubin, R.M. \& McAuliffe, M.D. (2008). The relation between childhood aggression and depressive symptoms: The unique and joint mediating roles of peer rejection and peer victimization. MerrillPalmer Quarterly, 54(3), 316-340. doi: 10. 1353/mpq.0.0000.

Ortega, R. \& Monks, C. (2005). Agresividad injustificada entre preescolares [Unjustified aggression among pre-schoolers]. Psicothe$m a, 17(3), 453-458$.

Palmer, A.L. (2011). Análisis de datos en el diseño unifactorial de medidas repetidas [Analysis of data in the repeated measures design unifactorial]. Madrid: La Muralla.

Phillips, B.M. \& Lonigan, C.J. (november, 2002). Multimethod replication of the tripartite model of anxiety and depression in a youth sample. Trabajo presentado en el Annual Meeting of the Association for the Advancement of Behavior Therapy, Reno.
Pössel, P., Seemann, S. \& Hautzinger, M. (2008). Impact of comorbidity in prevention of adolescent depressive symptoms. Journal of Counseling Psychology, 55(1), 106-117.

Quiggle, N.L., Garber, J., Panak, W.F. \& Dodge, K.A. (1992). Social information processing in aggressive and depressed children. Child Development, 63(6), 1305-1320. doi: 10. 2307/ 1131557.

Rowe, R., Maughan, B. \& Eley, T.C. (2006). Links between antisocial behavior and depressed mood: The role of life events and attributional style. Journal of Abnormal Child Psychology, 34(3), 293-302.

Rowe, R., Rijsdijk, F.V., Maughan, B., Hosang, G.M. \& Eley, T.C. (2008). Heterogeneity in antisocial behaviours and comorbidity with depressed mood: A behavioural genetic approach. Journal of Child Psychology and Psychiatry, 49(5), 526-534.

Rudolph, K.D. \& Clark, A.G. (2001). Conceptions of relationships in children with depressive and aggressive symptoms: Social-cognitive distortion or reality? Journal of Abnormal Child Psychology, 29(1), 41-56. doi: 10.1023/A:10 05299429060.

Saylor, C.F., Finch, A.J., Jr., Furey, W., Baskin, C.H. \& Kelly, M.M. (1984). Construct validity for measures of childhood depression: Application of multitrait-multimethod methodology. Journal of Consulting and Clinical Psychology, 52(6), 977-985.

Seeley, J.R. (2002). Comorbidity between conduct disorder and major depression: Phenomenology, correlates, course, and familial aggregation. Dissertation Abstracts International Section A: Humanities and Social Sciences, 62(12-A), 4122.

Serrano, G., Godás, A., Rodríguez, D. \& Mirón, L. (1996). Perfil psicosocial de los adolescentes españoles [Psychosocial characteristics of Spanish adolescents]. Psicothema, 8 (1), 25-44. 
Steinberg, L. \& Morris, A.S. (2001). Adolescent development. Annual Review of Psychology, 52, 83-110.

Subbarao, A., Rhee, S.H., Young, S.E., Ehringer, M.A., Corley, R.P. \& Hewitt, J.K. (2008). Common genetic and environmental influences on major depressive disorder and conduct disorder. Journal of Abnormal Child Psychology, 36(3), 433-444.

Teesson, M., Degenhardt, L., Proudfoot, H., Hall, W. \& Lynskey, M. (2005). How common is comorbidity and why does it occur? Australian Psychologist, 40(2), 81-87. doi: 10.1080/000 50060500094605.

Wakefield, J.C., Schmitz, M.F. \& Baer, J.C. (2010). Does the DSM-IV clinical significance criterion for major depression reduce false positives? Evidence from the National Comorbidity Survey Replication. American Journal of Psychiatry, 167(3), 298-304. doi: 10.1 176/ appi.ajp.2009.09040553.

Warrick-Swansen, S.A. (1999). Risk factors of comorbidity between aggressive behaviors and depressive disorders in school-aged children. Dissertation Abstracts International: Section B: The Sciences and Engineering, 59 (7-B), 3742.

Watson, D., Weber, K., Assenheimer, J.S., Clark, L.A., Strauss, M.E. \& McCormick, R.A. (1995). Testing a tripartite model: I. Evaluating the convergent and discriminant validity of anxiety and depression symptom scales. Journal of Abnormal Psychology, 104(1), 314.
Weersing, V.R. \& Weisz, J.R. (2002). Community clinic treatment of depressed youth: Benchmarking usual care against CBT clinical trials. Journal of Consulting \& Clinical Psychology, 70(2), 299-310.

Weiss, B. \& Catron, T. (1994). Specificity of the comorbidity of aggression and depression in children. Journal of Abnormal Child Psychology, 22(3), 389-401. doi: 10.1007/BF02168 081.

Weiss, B., Süsser, K. \& Catron, T. (1998). Common and specific features of childhood psychopathology. Journal of Abnormal Psychology, 107(1), 118-127.

Wolff, J.C. \& Ollendick, T.H. (2006). The comorbidity of conduct problems and depression in childhood and adolescence. Clinical Child and Family Psychology Review, 9(3), 201-220. doi: 10.1007/s10567-006-0011-3.

Ximénez, C. \& San Martín, R. (2000). Análisis de varianza con medidas repetidas [Analysis of variance with repeated measures]. Madrid: La Muralla.

Yang, J., Hong, S.D., Joung, Y.S. \& Kim, J. (2006). Validation study of Tripartite Model of anxiety and depression in children adn adolescents: Clinical sample in Korea. Journal Korean Medical Science, 21, 1098-1102. Youngstrom, E.A., Findling, R.L. \& Calabrese, J.R. (2003). Who are the comorbid adolescents? Agreement between psychiatric diagnosis, youth, parent, and teacher report. Journal of Abnormal Child Psychology, 31(3), 231-245. doi: 10.1023/a:1023244512119.
Universidad a Distancia de Madrid (UDIMA) Universidad Nacional de Educación a Distancia (UNED) Madrid - España

Fecha de recepción: 14 de noviembre de 2011 Fecha de aceptación: 9 de abril de 2012 
